\title{
THE EFFECTS OF VIEWPOINT ON THE VIRTUAL SPACE OF PICTURES
}

\author{
H. A. Sedgwick \\ Schnurmacher Institute for Vision Research \\ S.U.N.Y. College of Optometry, New York, New York
}

\section{INTRODUCTION}

Pictures are made for many different purposes (Hagen, 1986; Hochberg, 1979). This discussion is about pictorial displays whose primary purpose is to convey accurate information about the three-dimensional spatial layout of an environment. We should like to understand how, and how well, pictures can convey such information. I am going to approach this broad question through another question that seems much narrower. We shall find, however, that if we could answer the narrow question, we should have made a good start on answering the broader question as well.

Every pictorial display that presents a precise perspective view of some three-dimensional scene has a single geometrically correct viewpoint . ${ }^{1}$ In most viewing situations, however, the observer is not constrained to place his or her eye precisely at this correct viewpoint; indeed the observer generally has no explicit knowledge of the location of this viewpoint. ${ }^{2}$ My "narrow" question is: "What effect does viewing a picture from the wrong location have on the virtual space represented by that picture?"

This question is in itself of theoretical as well as practical importance. It has received considerable attention, but its answer is still far from being clear. The research literature is fragmentary and conflicting. I believe that a more vigorously applied theoretical analysis can clarify the issues and can help in evaluating the existing literature.

My theoretical analysis follows the approach developed by J. J. Gibson $(1947,1950$, $1954,1960,1961,1971,1979)$. I shall be referring frequently to the optic array, which is Gibson's term for the structured array of light reflected to a point of observation by the surfaces of the environment. I shall also be relying on Gibson's concept of available visual information. Information is said to be available in the optic array when some projective structure in the optic array mathematically specifies, with appropriate constraints, some structure in the environment. The optic array typically contains multiple, redundant sources of information for the spatial layout of the environment.

The theoretically determined availability of visual information of course does not guarantee that such information will be used by a human observer. The extent to which any such information actually influences perception is a separate question that must be addressed empirically. The contention of Gibson's approach is simply that we are not in a proper position to formulate or interpret empirical investigations of human visual perception until we understand the underlying available information on which any successful perception must be based.

This discussion will concentrate on theoretical analysis. At several points, however, I shall briefly indicate how well this analysis accords with the empirical work that has been done on 
human pictorial perception. More detailed reviews of this subject are offered elsewhere (Cutting, 1986a; Farber and Rosinski, 1978; Hagen, 1974; Kubovy, 1986; Rogers, 1985; Rosinski and

Farber, 1980).

To simplify the discussion I am going to consider separately the effects of deviating from the correct viewpoint in each of three orthogonal directions: deviations perpendicular to the picture plane (that is, being too close or too far from the picture), lateral deviations parallel to the picture plane, and vertical deviations parallel to the picture plane. Any possible viewing position can then be interpreted as some combination of these three deviations.

\section{THEORETICAL ANALYSIS}

\subsection{Viewing from Too Close or Too Far}

What is the theoretical effect of viewing a pictorial display from too close or too far ${ }^{3}$ ? As we approach or withdraw from the picture, its projection in the optic array expands or contracts around the center of the picture, which is the point at which a perpendicular from the viewpoint pierces the picture plane. If we let $z$ be the correct distance from the picture and $z^{\prime}$ be our actual distance, and let $\mathrm{A}$ and $\mathrm{A}^{\prime}$ be the angular separations from the center at these two distances, respectively, of some other point on the picture, then

$$
\tan A / \tan A^{\prime}=z^{\prime} / z=m
$$

where $\mathrm{m}$ is a constant. Thus the optic array projection of the picture is magnified or minified by $1 / \mathrm{m}$, where $\mathrm{m}$ measures how close or how far we are, relative to the correct distance. ${ }^{4}$

What, in theory, is the effect on the virtual space of the picture of magnifying or minifying its projection in the optic array? We can begin to answer this question by looking at the available visual information that is present in the perspective structure of the optic array, by which I mean the vanishing points of straight edges in the environment and the vanishing lines of planar
surfaces. 5

Let us imagine a picture of a flat, endless ground plane covered with a regular texture represented by a grid of lines. The horizon, or vanishing line, of the ground, will be located at eye level on the picture plane. If our point of observation is located at a height $h$ above the ground, then the distance $\mathrm{d}$ along the ground to any particular grid line parallel to the picture plane is given by the simple expression

$$
d=h(1 / \tan G)
$$

where $\mathrm{G}$ is the optic array angle subtended between the horizon of the ground plane and the grid line.

We can now combine these two expressions to derive the theoretical efiecl of magnification or minification. If we let $d^{\prime}$ be the geometrically specified distance of the grid line when the picture is seen from the incorrect viewpoint and let $G^{\prime}$ be the new optic array angle corresponding to 


$$
d^{\prime}=h\left(1 / \tan G^{\prime}\right)
$$

substituting for $\mathrm{G}^{\prime}$,

$$
d^{\prime}=h(m / \tan G)
$$

and substituting again,

$$
\mathrm{d}^{\prime}=\mathrm{md}
$$

Next, if we let $s$ be the specified separation in depth between any two successive grid lines, at distances $\mathrm{d} 1$ and $\mathrm{d} 2$, when the picture is seen from the correct viewpoint and let $\mathrm{s}^{\prime}, \mathrm{d} 1$ ', and $\mathrm{d} 2$ ' be the specified separation and distances when seen from the incorrect viewpoint, then

$$
\begin{aligned}
\mathrm{s}^{\prime} & =\mathrm{d} 2^{\prime}-\mathrm{d} 1^{\prime} \\
& =\mathrm{md} 2-\mathrm{md} 1 \\
& =\mathrm{m}(\mathrm{d} 2-\mathrm{d} 1) \\
& =\mathrm{ms}
\end{aligned}
$$

Thus as we approach the picture, the geometrically specified depths in the picture are compre ssed proportionally to the closeness of our approach and as we move away from the picture, depths are expanded proportionally (fig. 1). ${ }^{6}$

Consider now what happens to frontal plane dimensions. The tangent of the angle $\mathrm{F}$ subtended by a width $\mathrm{w}$ that is parallel to the picture plane is inversely proportional to its distance from the point of observation (assuming for simplicity that the width is measured from the center of the picture)

$$
\mathrm{w}=\mathrm{d} \tan \mathrm{F}
$$

As we approach the picture, the specified distance of $w$ decreases, but its optic array angle $F$ increases in the same proportion, so that $w$ remains constant (fig. 2)

$$
w^{\prime}=d^{\prime} \tan F^{\prime}=(m d)(\tan F / m)=d \tan F=w
$$

The depth of the pictured scene is thus compressed relative to its frontal dimensions. Shapes that are not in the frontal plane are distorted. The square grid covering the ground plane, for example, becomes a grid of rectangles whose depth to width ratio is $\mathrm{m}$ (fig. 3 ).

We may note here that all distances specified in the virtual space of the picture depend on $\mathrm{h}$, the height of the viewpoint above the ground plane, which thus provides a scale factor for all distances, as well as sizes, in the picture. Because $h$ itself is not geometrically specified in the picture, its value may be indeterminate. ${ }^{7}$ This indeterminancy of $h$ puts in doubt the appropriateness of comparing absolute distances or sizes across different pictures or across different views of the same picture. The ratio, however, of depth to width, $s / w$ or $s^{\prime} / w^{\prime}$, does not depend on $h$; thus, geometrically specified compression of shape by the factor $\mathrm{m}$ is an invariant effect of too close viewing. 
Geometrically specified angles and orientations in the pictured scene are also changed by approaching the picture. This result follows directly from the compression that occurs, but it is instructive to derive the result in a different way.

Every set of parallel lines in the pictured scene has a vanishing point on the picture plane (lines parallel to the picture plane have their vanishing points at infinity on the picture plane). The three-dimensional orientation of a set of parallel lines is equal to the orientation of a line from the point of observation to their vanishing point. This very simple optic array relation specifies the pictured orientation of any edge once its vanishing point is known (Hay, 1974; Sedgwick, 1980).

Edges perpendicular to the picture plane hive their vanishing point at the center of the picture. As we approach the picture, every vanishing point except for the one at the center of the picture increases its optic array separation from the central vanishing point. Thus the specified orientations of all nonperpendicular edges move closer to being parallel to the picture plane. For example, a square ground plane grid oriented at $45^{\circ}$ to the picture plane becomes a grid of squashed diamonds (fig. 4).

If we let $E$ be the angle, measured relative to the straight-ahead, that a vanishing point subtends at the correct viewpoint, and let $E^{\prime}$ be the angle that it subtends when the viewpoint is too close or too far, then the distortion $D$ in the specified orientation of edges having that vanishing point is given by $E$ minus $E^{\prime}{ }^{8}$ The relation between $E$ and $E^{\prime}$ is the same as for any other optic array angles measured from the center of the picture, namely

$$
\tan E / \tan E^{\prime}=m
$$

Calculating $D$ as a function of $E$ for several values of $m$, we obtain a family of curves showing no distortion for orientations perpendicular $\left(0^{\circ}\right)$ or parallel $\left(-90^{\circ}\right.$ or $\left.90^{\circ}\right)$ to the picture plane, with maximum distortion at intermediate values (fig. 5). For example, for $\mathrm{m}$ equal to either 2 or 0.5 , the maximum distortion approaches $20^{\circ}$.

A similar analysis can be made for the orientations of planar surfaces. The angle subtended between the vanishing line of a slanted surface and the vanishing line of the ground plane is equal to the three-dimensional angle between the depicted surface and the ground (Sedgwick, 1980). As we approach the picture plane, geometrically specified surface orientations are distorted in just the same way as are edge orientations.

Perceptually, effects qualitatively similar to those predicted theoretically here can be seen by a careful observer moving closer or farther from a picture containing strong linear perspective. If the perspective information in the picture is weaker, the distortions may be much harder to see. Most empirical investigations, but not all, have found such distortions in human picture perception, although not always at the magnitude predicted. 9 I shall say a bit more about the reasons for the discrepancies between investigations later.

\subsection{Viewing from the Side}

Let us now consider what happens when we view a pictorial display from the side. 10 It is easy to see that when the viewpoint is displaced laterally, maintaining the same distance from the picture plane, the horizon of the ground and all of the grid lines parallel to the picture plane simply 
slide along themselves in the optic array. Thus the angular separation of each of these grid lines from the ground horizon remains unchanged. Consequently, the geometrically specified distance of each of these grid lines, relative to the height of the viewpoint, also is unchanged (fig. 6).

As the viewpoint slides to the right, for example, each point in the geometrically specified virtual space of the picture slides to the left, with its projected point on the surface of the picture acting as a stationary fulcrum. This lateral shift in virtual space is thus directly proportional to, but opposite in sign from, the amount of the viewpoint's displacement; it is also directly proportional to the distance of the point from the picture plane, and is inversely proportional to the viewpoint's distance from the picture plane (fig. 7). The overall effect of this viewpoint displacement is to produce a lateral shear in the geometrically specified virtual space of the picture (fig. 8). Frontal plane dimensions and orientations are unchanged, but shapes and orientations extending in depth are all distorted.

We can readily determine the specified shifts in the orientations of pictured edges and surfaces by again making use of the perspective structure of the picture. Let us consider, as an example, the orientations of horizontal edges, whose vanishing points lie on the horizon of the ground plane. As the viewpoint shifts laterally, its angular relation to each of these vanishing points changes. We shall let $\mathrm{E}$ again be the angle, measured relative to the straight-ahead, that the vanishing point makes with the correct viewpoint, and let $E^{\prime}$ be the angle that it makes after the vanishing point has shifted laterally. We can express this lateral shift as the ratio, $k$, between the amount, $r$, of the shift, and the distance, $z$, of the viewpoint from the picture plane. It is easy to see that (fig. 9)

$$
\tan E^{\prime}=\tan E+k
$$

If we express the position of the shifted viewpoint in terms of its angular deviation, $V$, from the correct viewpoint, then

$$
\tan V=k
$$

so that

$$
\tan E^{\prime}=\tan E+\tan V
$$

We can use this relation to determine the specified distortion of orientation, E' minus $E$, as a function of the correct orientation $\mathrm{E}$, for a variety of angular shifts $\mathrm{V}$ of the viewpoint (fig. 10). The resulting family of curves shows that the specified distortions in orientation can be very large, approaching $180^{\circ}$ as $\mathrm{V}$ approaches $\pm 90^{\circ}$, which is parallel to the picture plane, and that the orientation $\mathrm{E}$ at which the distortion is maximal increases as $\mathrm{V}$ increases.

We may note that the same distortions in orientation would also be specified for vertical planes in the virtual space of the picture when the viewpoint is displaced laterally.

Perceptually, again, a careful observer comparing the appearance of a picture seen from one side or the other can notice differences in apparent orientation if the picture contains sufficient perspective information. Some empirical investigations have also found results that are qualitatively similar to those derived here, although others have not. ${ }^{11}$ Again, I shall refer back to these discrepancies a little later. 


\subsection{Viewing from Too High or Too Low}

Let us now briefly consider what happens when the viewpoint is too high or too low. This is again a displacement parallel to the picture plane, so the geometrically specified distortions in the virtual space of the picture are identical in form to those produced by lateral shifts, except that here the virtual space is sheared vertically instead of laterally.

Thus, for example, if we consider a plane in virtual space that is rotated around a horizontal axis so that it makes an angle $E$ with the ground, its specified slant $E^{\prime}$, when seen from an incorrect viewpoint having a vertical angular deviation $\mathrm{V}$, is given by the same relation

$$
\tan E^{\prime}=\tan E+\tan V
$$

Notice that if we are considering the ground plane itself, then $E=0$, so that $E^{\prime}=V$. That is, if we must look down by a certain angle to see the pictured horizon, then the ground plane is specified as slanting down by that same angle.

\section{THEORETICAL COMPLICATIONS}

So far we have seen how we can use the perspective structure of the optic array to determine the geometrically specified sizes, distances, and orientations of surfaces and edges in the virtual space of a picture. We have also seen how this visual information, when it is present, specifies distortions in the pictured layout when we observe the picture from the wrong viewpoint. Unfortunately for our ease of understanding, there are theoretical complications that are not taken into account by this straightforward analysis. We need to consider some of these complications now.

\subsection{Resolving Multiple Sources of Visual Information}

In a normally complex pictorial display, there are available other sources of visual information for spatial layout besides those arising from the perspective structure of the picture. How these multiple sources of information, which are normally partially redundant and partially complementary, may be combined into a single perceptual interpretation is a difficult and as yet unsettled question. ${ }^{12}$ The difficulty is increased when the picture is observed from the wrong viewpoint because these different sources of information do not all predict the same distortions; nor is it always easy to tell what they do predict.

As an example, consider some of the information arising from surface texture (Gibson, 1950; Sedgwick, 1983, 1986). If several edges are resting on a surface that is uniformly textured, then the relative lengths of the edges are specified by the relative amounts of texture that they cover; likewise, the relative distances between the edges are specified by the relative amounts of texture between them. This texture scale information is as valid for edges that extend into depth as for those in the frontal plane; it thus serves to specify the shapes and the relative sizes and distances of objects resting on a common textured surface such as the ground plane. 
It is easy to see that all such texture scale information is completely invariant over changes in viewpoint because such changes do nothing to alter the depicted amounts of texture between or under the objects in the picture. If, for example, we approach the picture of a square object resting on the textured ground, the specified object remains square because each of its edges continues to cover an equal amount of texture. On the other hand, according to the analysis based on perspective structure, the specified object is compressed into a rectangle whose width is greater than its depth.

This apparent contradiction between the distortions predicted by these two sources of visual information can be resolved, but only in a way that further complicates our analysis. I mentioned earlier that any visual information entails constraints on the environment; if these constraints are violated, then the information is no longer valid. In the case of texture scale information, an essential constraint is that the texture's distribution across a surface be at least statistically uniform. Yet, in the example that we are considering now, when we come too close to the picture, perspective analysis specifies that the texture of the ground is itself compressed in the depth dimension. Thus the uniform distribution constraint is violated and texture scale information is no longer valid.

A visual system might do any of a number of things when faced with this situation. It might simply reject texture scale information as being invalid. It might go ahead and use texture scale information anyway. It might recognize that the viewpoint is incorrect. It might abandon the attempt to find a consistent virtual space for the picture. It might adopt a modified version of texture scale information using compressed texture. It might do something intermediate between some of these options. Analysis only indicates the possibilities without specifying which one will be adopted by any particular visual system.

A number of other sources of visual information, such as right-angle constraints (Perkins, 1972, 1976) and orientation-distribution constraints (Witkin, 1980), present similar difficulties when the viewpoint is incorrect, but there is not space to consider these additional difficulties here. Careful analysis of the interactions between these different sources of information should give us a basis for manipulating the information content of pictures so as to better determine the perceptual effects they produce.

\subsection{Constancy and the Dual Nature of Pictures}

A second set of theoretical complications arises from what has often been referred to as the "dual nature" of pictures (Gibson, 1954; Haber, 1979, 1980a, 1980b; Hagen, 1974, 1986; Hochberg, 1962, 1979; Pirenne, 1970). In addition to being a representation of a spatial layout existing in a three-dimensional virtual space that lies beyond the plane of the picture, a pictorial display is also a real object consisting of markings of some sort, usually on a flat surface. Normally, visual information for the flat surface of the picture is made available by binocular stereopsis, by motion parallax, by the oculomotor adjustments of convergence and accommodation, by the frame of the picture, and by the surface texture of the picture.

To perceive pictures, a perceptual system must be able, to some extent, to differentiate its response to the picture's virtual layout from its response to the real layout of the picture's surface. The human visual system seems able to make this differentiation, but not without some interaction, or "cross talk," between its responses to these two classes of information. 
We can get some understanding of one effect of the picture surface by examining the relation between the picture plane and the optic array. If $x$ measures a separation in the picture plane from the center of the picture, which we have already defined as the point where a perpendicular from the viewpoint pierces the picture plane, and A measures the optic array angle subtended by this separation, then $\mathrm{x}$ is related to $\mathrm{A}$ by the relation

$$
\mathrm{x}=\mathrm{z} \tan \mathrm{A}
$$

where $z$ is the distance from the viewpoint to the picture plane. Near the center of a picture there is a close congruence between the optic array projection and the flat picture plane projection. This is because the tangent function is nearly linear for small angles. For larger angles, however, the tangent function becomes highly nonlinear, and consequently the optic array projection and the picture plane projection become strongly noncongruent.

Perceptually, the cross talk between the picture surface and the virtual space of the picture, as specified in the optic array, becomes most noticeable when the picture plane projection and the optic array projection are noncongruent. Toward the edges of wide-angle pictorial displays, for example, the projections on the picture plane and in the optic array are still geometrically correct, but objects in the virtual space of the picture often appear to be distorted (Pirenne, 1970, 1975; Kubovy, 1986). ${ }^{13}$ It seems that the noncongruent shape on the surface of the picture takes on a perceptual salience that interacts with the virtual space of the picture.

A similar noncongruence between the picture plane and the optic array is produced when the viewpoint is displaced laterally or vertically from the correct viewpoint. Again, the noncongruent shape on the surface of the picture may interact perceptually with the virtual space of the picture, but here its effect would be to diminish the distortion that is specified in the optic array. This would result in some degree of "constancy" in the virtual space of the picture in the sense that the virtual layout would not be as distorted as the optic array information would predict.

These effects of the picture's surface on the perceived virtual space of the picture could be eliminated, in principle, by removing the visual information for the picture's surface. Using a monocular display, restricting head movements relative to it, hiding the frame of the display, and so on, would all contribute to this result (Ames, 1925; Enright, 1987; Schlosberg, 1941; P. C.

Smith and O. W. Smith, 1961).

\subsection{The Hypothesis of Pictorial Compensation}

Finally, many theorists have suggested that when information for the picture surface is available, the human visual system may be able to compensate for being at the wrong viewpoint and so avoid distortions in the virtual space of the picture (Cutting, 1987; Farber and Rosinski, 1978; Hagen, 1974, 1976a, 1976b; Kubovy, 1986; Perkins, 1973, 1980; Pirenne, 1970; Rosinski, 1976; Rosinski and Farber, 1980; Rosinski, Mulholland, Degelman, and Farber, 1980; Wallach and Marshall, 1986). This compensation process would operate by either detecting or assuming a "correct" position of the viewpoint. The optic array information would then be adjusted to determine the virtual layout as it would be seen from this correct viewpoint.

Although a number of experiments have been offered in support of this view, it seems to me that, on balance, the compensation hypothesis is neither necessary nor sufficient to account for 
the bulk of the empirical results. It is not necessary because, as we have just seen, however sketchily, there are other explanations available for some of the disparities that exist between the distortions predicted by perspective structure and those actually found. Moreover, these other explanations are more parsimonious, in that they are derived from the analysis of general perceptual processes without having to postulate special processes that exist solely for perceiving pictures from the wrong viewpoint. The compensation hypothesis is not sufficient because it does not account for the considerable number of experimental results that find distortions in virtual space even when there is information available for the surface of the picture (Bengston, et al., 1980; Goldstein, 1979, 1987; Wallach, 1976, 1985). Finally, it seems to me that a careful reading of several of the key experiments offered in favor of the compensation hypothesis casts some doubt on the firmness of their conclusions. ${ }^{14}$

\section{CONCLUSION}

As a conclusion to this brief discussion, I would suggest that picture perception is not best approached as a unitary, indivisible process. Rather, it is a complex process depending on multiple, partially redundant, interacting sources of visual information for both the real surface of the picture and the virtual space beyond. Each picture must be assessed for the particular information that it makes available. This, I would suggest, will determine how accurately the virtual space represented by the picture is seen, as well as how it is distorted when seen from the wrong viewpoint. 


\section{NOTES}

1. For a camera image, this point is determined by the optics of the imaging system; for a display created by a draftsman or a computer, this point is determined by the relation between the center of projection and the projection plane (Carlbom and Paciorek, 1978; Sedgwick, 1980).

2. A complex pictorial display generally does contain sufficient information, under certain constraints, to specify its own correct viewpoint. This issue is discussed by Green (1983), Jones and Hagen (1978), and Sedgwick (1980).

3. A number of analyses of this problem have been offered. The first systematic analysis appears to come from La Gournerie (1859), whose work has been discussed more recently by Pirenne (1970, 1975), Kubovy (1986), and Cutting (1987). Other analyses, apparently independent of La Gournerie, have been given by Purdy (1960), Farber and Rosinski (1978), Lumsden (1980), and Rosinski and Farber (1980).

Obtaining an unambiguous three-dimensional interpretation of a pictorial display requires that some constraints be placed on the possible interpretations. In the above analyses, those referring to La Gournerie and that of Farber and Rosinski (1978) do not make these constraints explicit. The other analyses use explicit constraints derived from analyses of normally viewed pictures. Purdy (1960) bases his analysis on gradients of texture, Lumsden bases his on familiar size, and Rosinski and Farber base theirs on linear perspective. I offer two analyses here, one based on the ground plane and the other based on perspective structure, as suggested in Sedgwick (1980). All of these analyses converge on the same results.

A different analysis, reaching different results, has been offered recently by McGreevy and his colleagues (Ellis et al., 1985; McGreevy and Ellis, 1984, 1986; McGreevy, Ratzlaff, and Ellis, 1987). McGreevy's analysis proceeds by arbitrarily constraining all virtual distances from the picture plane to be unchanged by viewing position. This analysis has the weakness that it assumes a knowledge of these distances without indicating how they could be determined by an observer of the display, either when viewing from the wrong viewpoint or when viewing from the correct viewpoint. The question of how virtual layout could be determined here is made difficult because the constraint that is imposed leads to violations of all of the other constraints mentioned in the preceding paragraph.

Another kind of analysis, based on optimizing the match between a noisy registration of the projection and a noisy a priori internal model of the spatial layout has been offered recently by Grunwald and Ellis (1986). There is not room here to consider the interesting question of how such a model-based approach to spatial layout might be reconciled with the constraint-based approach taken in this paper.

4. Approaching a picture is optically equivalent to viewing the pictured scene through a telephoto lens, and withdrawing from the picture is optically equivalent to viewing the scene through a wide-angle lens (Lumsden, 1980; Rosinski and Farber, 1980).

5. Perspective structure is usually only implicit in the optic array. The available visual information that specifies this perspective structure is not discussed in this paper, but I have analyzed it 
in detail elsewhere. Not all pictorial displays contain sufficient information to completely specify their perspective structure (Sedgwick, 1983, 1986, 1987a).

6. There is an invariant associated with the optic array gradient projected from equally spaced grid lines parallel to the picture plane. If $s$ is the separation in depth between any two successive grid lines, then

$$
\mathrm{s}=\mathrm{d} 2-\mathrm{d} 1=\mathrm{h}(1 / \tan \mathrm{G} 2-1 / \tan \mathrm{G} 1)
$$

Thus, for any two successive optic array angles G1 and G2 in this gradient

$$
1 / \tan \mathrm{G} 2-1 / \tan \mathrm{G} 1=\mathrm{k}
$$

where $\mathrm{k}$ is a constant. The presence of this invariant in the optic array specifies that the grid lines are equally spaced. It can be shown that this invariant is preserved when the picture is viewed from too close or too far.

7. The value of $\mathrm{h}$ can be determined by assuming that the ground plane of the picture is coextensive with the ground plane of the real environment, but such an assumption may for some pictures be neither appropriate nor perceptually compelling.

8. Throughout this paper, orientations are specified in environment-centered terms (i.e., relative to the fixed framework of the environment), rather than in viewer-centered terms (i.e., relative to the observer's line of regard). I have discussed this distinction and its significance at length elsewhere (Sedgwick, 1983; Sedgwick and Levy, 1985).

9. Empirical evidence that is at least qualitatively consistent with the analysis presented here has been reported by Bartley (1951), Bartley and Adair (1959), Bengston et al. (1980), Farber (1972), Lumsden (1983), Purdy (1960), O. W. Smith (1958a, 1958b), O. W. Smith and Gruber (1958), and O. W. Smith, P. C. Smith, and Hubbard (1958). Anecdotal supporting observations are also reported by MacKavey (1980) and Pirenne (1970). On the other hand, Rosinski and Farber (1980) briefly report failing to find distortions when the frame of the display is visible, and Hagen and Elliott (1976) and Hagen and Jones (1978) report that adults' choice of the most "realistic looking" display was essentially independent of their actual viewing distance.

It is important to distinguish between the presence of measurable distortions in the perception of spatial layout and the detection of these distortions by the observer. Observers' perceptions may contain distortions of which the observers themselves are unaware. A number of researchers have suggested that observers are often not very sensitive to the presence of such distortions (Gombrich, 1972; Pirenne, 1970; Cutting, 1986a, 1986b).

10. Systematic analysis of this problem is again offered by La Gournerie (1859), whose work has been put to use by Cutting (1987). More recent analyses are offered by Farber and Rosinski (1978) and Rosinski and Farber (1980), who explicitly base their second analysis (1980) on linear perspective constraints. I again offer two analyses, one based on the ground plane and the other, following Sedgwick (1980), based on perspective structure. All of these analyses agree in the distortions that they predict. 
11. Anecdotal reports of these distortions are common (Pirenne, 1970, 1975; Wallach, 1976, 1985). Experimental evidence that such distortions occur perceptually under some circumstances is offered by Goldstein $(1979,1987)$, Rosinski et al. (1980), Rosinski and Farber (1980), and Wallach and Marshall (1986), although all of these authors also report conditions under which the analytically predicted distortions do not occur. Cutting (1987) has analyzed some of the data of Goldstein (1987) in detail and has shown it to be in generally good accord with the theoretical predictions. Perkins (1973) finds some distortion from lateral viewing, but much less than this analysis would predict.

12. An expert system that I have developed to study the interaction of multiple sources of visual information is described elsewhere (Sedgwick, 1987a, 1987b).

13. This assumes that the perpendicular from the correct viewpoint pierces the picture plane somewhere near the center of the pictorial display, as it usually does.

14. Kubovy (1986) is critical of many of the stimuli used by Hagen and Elliott (1976) and Hagen and Jones (1978) in their demonstration that adults at various distances from a picture do not choose the correct perspective as being most realistic. Perkins' (1973) demonstration of compensation for lateral viewing uses such minimal stimuli that the applicability of his results to more complex displays may reasonably be questioned. Hagen's (1976b) study, which claims to find evidence of compensation for lateral viewing in adults, has been criticized at length on logical grounds by Rogers (1985), who also failed to replicate Hagen's results. In the carefully controlled study of Rosinski et al. (1980) on the effects of frame visibility on perceived surface slant with lateral viewing, the interpretation of results is clouded by a confusion in the description of the experiment, and possibly in the experiment itself, about the frame of reference for their observers' judgments. Finally, Wallach and Marshall $(1986$, exp. 2) find evidence of compensation in pictorial shape perception from a lateral viewpoint, but their results, as they note, could be due to ordinary shape constancy because their stimulus shape was nearly parallel to the picture plane. 


\section{REFERENCES}

Ames, A. The illusion of depth from single pictures. J. Opt. Soc. Amer., 1925, 10, 137-148.

Bartley, S. H. A study of the flattening effect produced by optical magnification. Amer. J. Optom., 1951, 28, 290-299.

Bartley, S. H., and Adair, H. J. Comparisons of phenomenal distance in photographs of various sizes. J. Psychol., 1959, 47, 289-295.

Bengston, J. K., Stergios, J. C., Ward, J. L., and Jester, R. E. Optic array determinants of apparent distance and size in pictures. LExp. Psychol. Human Percept. Perform., 1980, 6, 751-759.

Carlbom, I., and Paciorek, J. Planar geometric projections and viewing transformations. Computing Surv., 1978, 10, 465-502.

Cutting, J. E. Perception with an Eye for Motion. Cambridge, MA: Bradford Books/The MIT Press, 1986a.

Cutting, J. E. The shape and psychophysics of cinematic space. Behav. Res. Meth. Instrum. Computers, $1986 \mathrm{~b}, 18$, 551-558.

Cutting, J. E. Affine distortions of pictorial space: Some predictions for Goldstein (1987) that La Gournerie (1859) might have made. J, Exp. Psychol.: Human Percept. Perform., 1987 , in press.

Ellis, S. R., Tyler, M., Kim, W. S., McGreevy, M. W., and Stark, L. Visual enhancements for perspective displays: Perspective parameters. Proc. 1985 IEEE Intern. Conf. Systems. Man. and Cybernetics, Tucson, AZ, November 12-15, 1985, 815-818.

Enright, J. T. Perspective vergence: oculomotor responses to line drawings. Vision Res., 1987, 27, 1513-1526.

Farber, J. M. The effects of angular magnification on the perception of rigid motion (Doctoral dissertation, Cornell Univ., 1972).

Farber, J., and Rosinski, R. R. Geometric transformations of pictured space. Perception, 1978, 7, 269-282.

Gibson, J. J. Motion Picture Testing and Research (Army Air Forces Aviation Psychology Program Research Reports, No. 7). Washington, DC: GPO, 1947.

Gibson, J. J. The Perception of the Visual World. Boston: Houghton Mifflin, 1950.

Gibson, J. J. A theory of pictorial perception. Audio-Visual Commun. Rev., 1954, 2 , 3-23.

Gibson, J. J. Pictures, perspective, and perception. Daedalus, 1960, 89, 216-227. 
Gibson, J. J. Ecological optics. Vision Res., 1961, 1, 253-262.

Gibson, J. J. The information available in pictures. Leonardo, 1971, 4, 27-35.

Gibson, J. J. The Ecological Approach to Visual Perception. Boston: Houghton Mifflin, 1979.

Goldstein, E. B. Rotation of objects in pictures viewed at an angle: Evidence for different properties of two types of pictorial space. J. Exp. Psychol.: Human Percept. Perform., 1979, $5,78-87$.

Goldstein, E. B. Spatial layout, orientation relative to the observer, and perceived projection in pictures viewed at an angle. LExp. Psychol. Human Percept. Perform., 1987, 13, 256-266.

Gombrich, E. H. The "what" and the "how": Perspective representation and the phenomenal world. In R. Rudner and I. Scheffler (Eds.), Logic and Art: Essays in Honor of Nelson Goodman. Indianapolis: Bobbs-Merrill, 1972.

Green, R. Determining the preferred viewpoint in linear perspective. Leonardo, 1983, 16, 97-102.

Grunwald, A. J., and Ellis, S. R. Spatial orientation by familiarity cues. Proc. 6th European Conf. Human Decision Making and Cont. Amsterdam: Elsevier Science Publications (in press).

Haber, N. H. Perceiving the layout of space in pictures: A perspective theory based upon Leonardo da Vinci. In C. F. Nodine and D. F. Fisher (Eds.) Perception and Pictorial Representation, Praeger, 1979, 84-89.

Haber, N. H. How we perceive depth from flat pictures. Amer. Scientist, 1980a, 68, 370-380.

Haber, N. H. Perceiving space from pictures: A theoretical analysis. In M. A. Hagen (Ed.), The Perception of Pictures, vol. 1. New York: Academic Press, 1980b, 3-31.

Hagen, M. A. Picture perception: Toward a theoretical model. Psychol. Bull., 1974, $\underline{81}$, 471-497.

Hagen, M. A. Problems with picture perception: A reply to Rosinski. Psychol. Bull., 1976a, $\underline{83}$, 1176-1178.

Hagen, M. A. Influence of picture surface and station point on the ability to compensate for oblique view in pictorial perception. Developmental Psychol., 1976b, 12, 57-63.

Hagen, M. A. Varieties of Realism: Geometries of Representational Art, Cambridge: Cambridge University Press, 1986.

Hagen, M. A., and Elliott, H. B. An investigation of the relationship between viewing condition and preference for true and modified linear perspective with adults. J. Exp. Psychol.i Human Percept. Perform., 1976, 2, 479-490. 
Hagen, M. A., and Jones, R. K. Differential patterns of preference for modified linear perspective in children and adults. J. Exp. Child Psychol., 1978, 26, 205-215.

Hay, J. C. The ghost image: A tool for the analysis of the visual stimulus. In R. B. MacLeod and H. L. Pick, Jr. (Eds.), Perception: Essays in Honor of James J. Gibson. Ithaca, NY: Cornell Univ. Press, 1974, 268-275.

Hochberg, J. E. Psychophysics of pictorial perception. Audio-Visual Commun. Rev., 1962, 10, 22-54.

Hochberg, J. E. Some of the things that paintings are. In C. F. Nodine and D. F. Fisher (Eds.) Perception and Pictorial Representation. Praeger, 1979, 17-41.

Jones, R. K., and Hagen, M. A. The perceptual constraints on choosing a pictorial station point. Leonardo, 1978, 10, 1-6.

Kubovy, M. The Psychology of Linear Perspective in Renaissance Art. New York: Cambridge Univ. Press, 1986.

La Gournerie, J. de. Traite de perspective lineaire contenant les traces pour les tableaux plans et courbes, les bas-reliefs, et les decorations theatrales, avec une theorie des effets de perspective. 1 vol. and 1 atlas of plates. Paris: Dalmont et Dunod; Mallet-Bachelier, 1859.

Lumsden, E. A. Perception of radial distance as a function of magnification and truncation of depicted spatial layout. Percept. Psychophys., 1983, 33, 177-182.

Lumsden, E. A. Problems of magnification and minification: An explanation of the distortion of distance, slant, shape, and velocity. In M. A. Hagen (Ed.), The Perception of Pictures, vol. 1, New York: Academic Press, 1980, 91-135.

Mackavey, W. R. Exceptional cases of pictorial perspective. In M. A. Hagen (Ed.), The Perception of Pictures, vol. 1, New York: Academic Press, 1980, 213-223.

McGreevy, M. W., and Ellis, S. R. Direction judgement errors in perspective displays. Proc. 20th Annual Conf. on Manual Contr., 1984, NASA CP 2341, 1, 531-549.

McGreevy, M. W., and Ellis, S. R. The effect of perspective geometry on judged direction in spatial information instruments. Human Factors, 1986, 28, 439-456.

McGreevy, M. W., Ratzlaff, C. R., and Ellis, S. R. Virtual space and two-dimensional effects in perspective displays (manuscript, 1987).

Perkins, D. N. Visual discrimination between rectangular and nonrectangular parallelopipeds. Percept. Psychophys., 1972, 12, 396-400.

Perkins, D. N. Compensating for distortion in viewing pictures obliquely. Percept. Psychophys., $1973,14,13-18$.

Perkins, D. N. How good a bet is a good form? Perception, 1976, 5, 393-406. 
Perkins, D. N. Pictures and the real thing. In P. A. Kolers, M. E. Wrolstad, and H. Bouma (Eds.) Processing of Visible Language 2. New York: Plenum, 1980, 259-278.

Pirenne, M. H. Optics, Painting, and Photography. Cambridge: Cambridge Univ. Press, 1970.

Pirenne, M. H. Vision and art. In E. C. Carterette and M. P. Friedman (Eds.) Handbook of Perception, vol. 5. Seeing. New York: Academic Press, 1975, 433-490.

Purdy, W. C. The hypothesis of psychophysical correspondence in space perception. Ithaca, NY: General Electric Advanced Electronics Center, September 1960 (NTIS R60ELC56).

Rogers, S. Representation and reality: Gibson's concept of information and the problem of pictures. (Doctoral dissertation, Royal College of Art, 1985).

Rosinski, R. R. Picture perception and monocular vision: A reply to Hagen. Psychol. Bull., $1976, \underline{83}, 1172-1175$.

Rosinski, R. R., and Farber, J. Compensation for viewing point in the perception of pictured space. In M. A. Hagen (Ed.), The Perception of Pictures, vol. 1, New York: Academic Press, 1980, 137-176.

Rosinski, R. R., Mulholland, T., Degelman, D., and Farber, J. Picture perception: An analysis of visual compensation. Percept. Psychophys., 1980, 28, 521-526.

Schlosberg, H. Stereoscopic depth from single pictures. Amer. J. Psychol., 1941, 54, 601-605.

Sedgwick, H. A. The geometry of spatial layout in pictorial representation. In M. A. Hagen (Ed.), The Perception of Pictures. vol. 1, New York: Academic Press, 1980, 33-90.

Sedgwick, H. A. Environment-centered representation of spatial layout: Available visual information from texture and perspective. In J. Beck, B. Hope, and A. Rosenfeld (Eds.), Human and Machine Vision. New York: Academic Press, 1983.

Sedgwick, H. A. Space perception. In K. Boff, L. Kaufman, and J. Thomas (Eds.), Handbook of Perception and Human Performance (Vol, 1). New York: Wiley, 1986.

Sedgwick, H. A. A production system modeling high-level visual perspective information for spatial layout. Technical Report No. 298, New York Univ. Depart. of Computer Sci., $1987 \mathrm{a}$.

Sedgwick, H. A. Layout2: A production system modeling visual perspective information. Proc. IEEE First Intern, Conf, on Computer Vision, London, June 8-11, 1987b.

Sedgwick, H. A., and Levy, S. Environment-centered and viewer-centered perception of surface orientation. Computer Vision. Graphics, and Image Processing, 1985, 31, 248-260.

Smith, $\mathrm{O}$. W. Comparison of apparent depth in a photograph viewed from two distances. Percept. Motor Skills, 1958a, 8, 79-81. 
Smith, O. W. Judgments of size and distance in photographs. Amer. J. Psychol., 1958b, 11, 529-538.

Smith, O. W., and Gruber, H. Perception of depth in photographs. Percept. Motor Skills, 1958, $\underline{8}$, 307-313.

Smith, O. W., Smith, P. C., and Hubbard, H. Perceived distance as a function of the method of representing perspective. Amer. J. Psychol., 1958, 71, 662-674.

Smith, P. C., and Smith, O. W. Ball throwing responses to photographically portrayed targets. J. Exp. Psychol., 1961, 62, 223-233.

Wallach, H. The apparent rotation of pictorial scenes. In M. Henle (Ed.) Vision and Artifact, New York: Springer, 1976, 65-69.

Wallach, H. Perceiving a stable environment. Scientific Amer., 1985, 252, 118-124.

Wallach, H., and Marshall, F. J. Shape constancy in pictorial representation. Percept. Psychophys., 1986, 39, 233-235.

Witkin, A. P. Shape from contour. MIT technical report AI-TR-589, November 1980. 


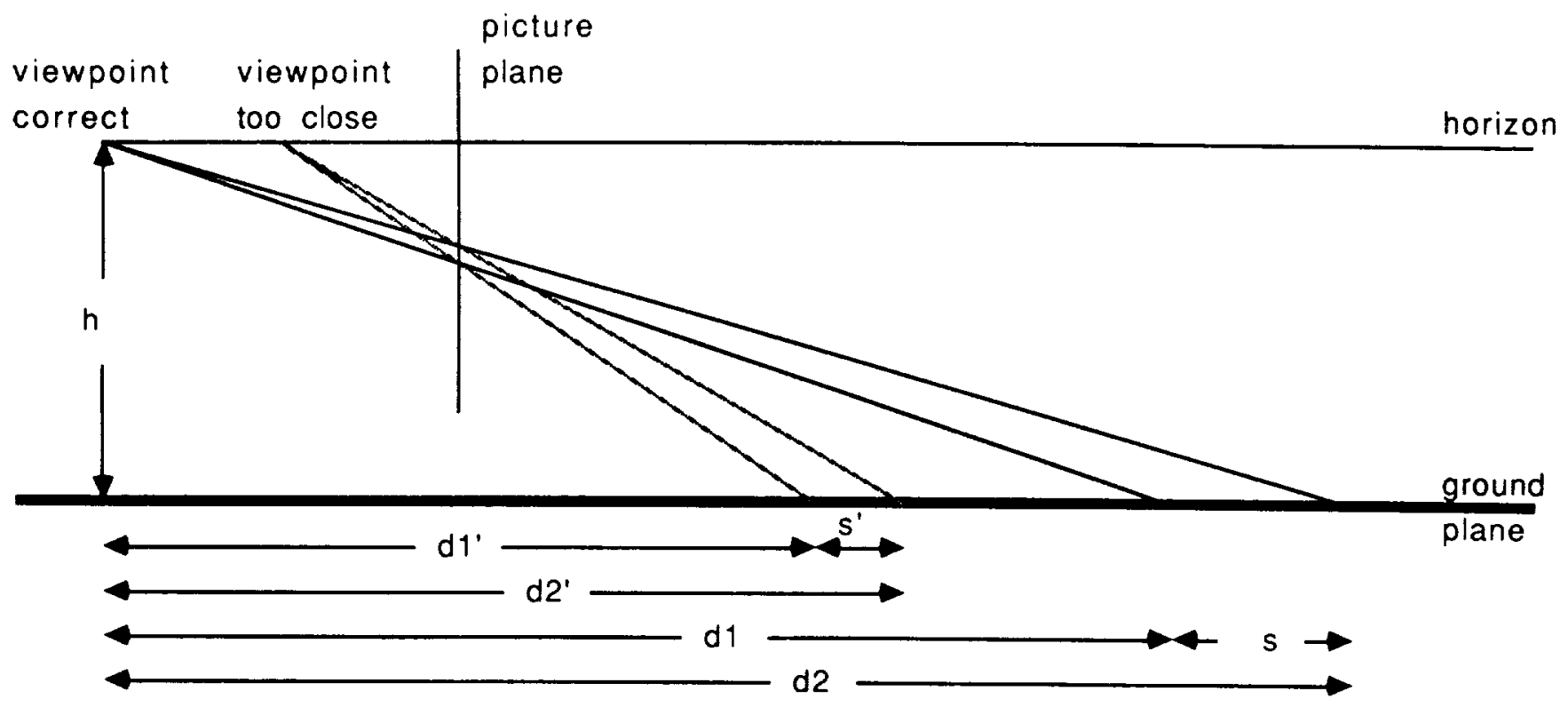

Figure 1.- Close viewing compresses geometrically specified virtual depth.

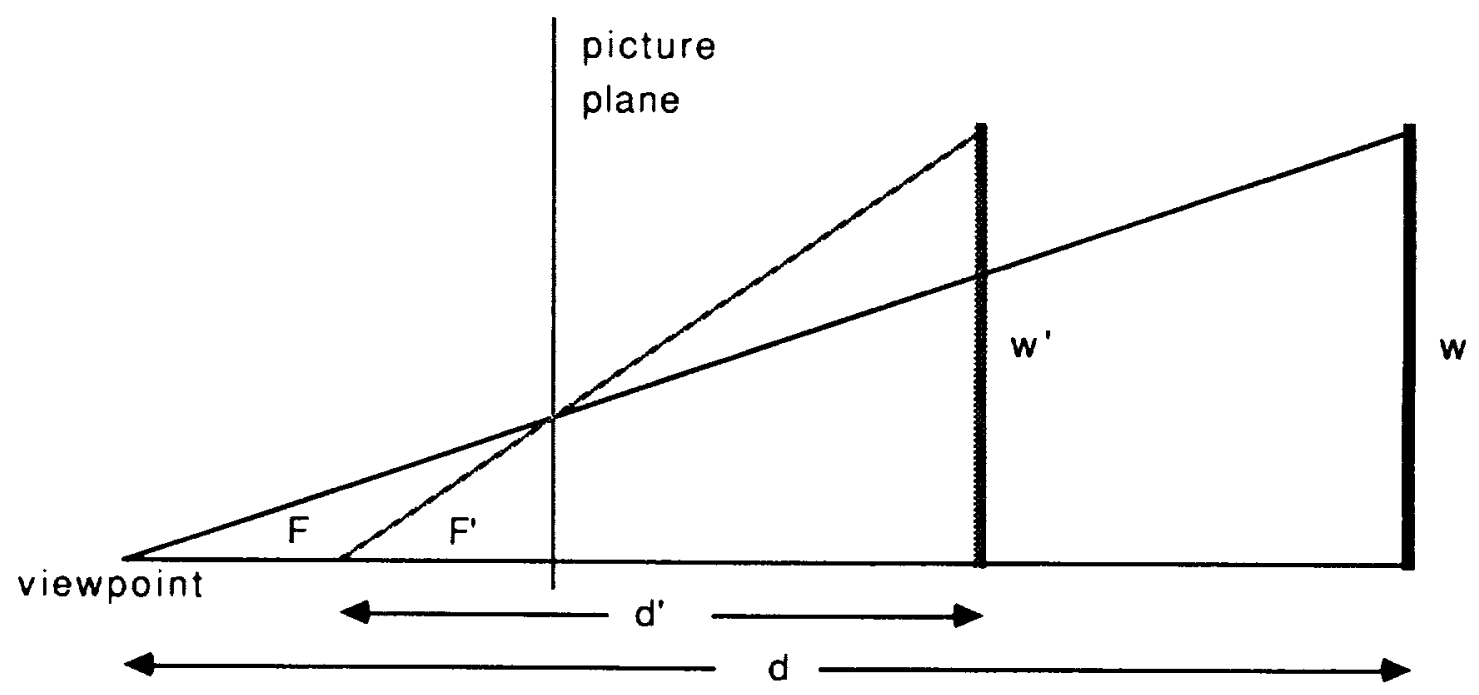

Figure 2.- Close viewing leaves geometrically specified virtual frontal dimensions unchanged. 


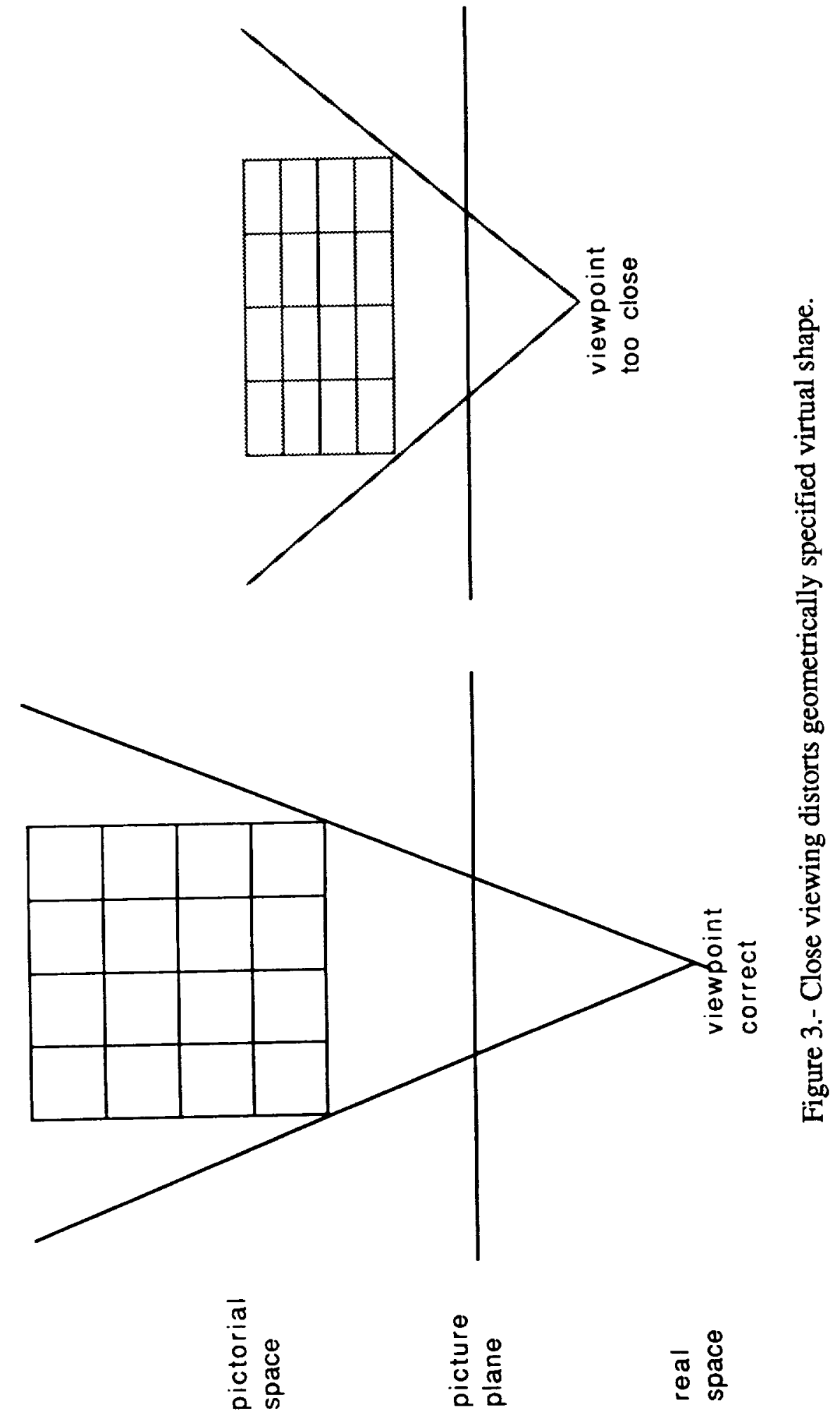




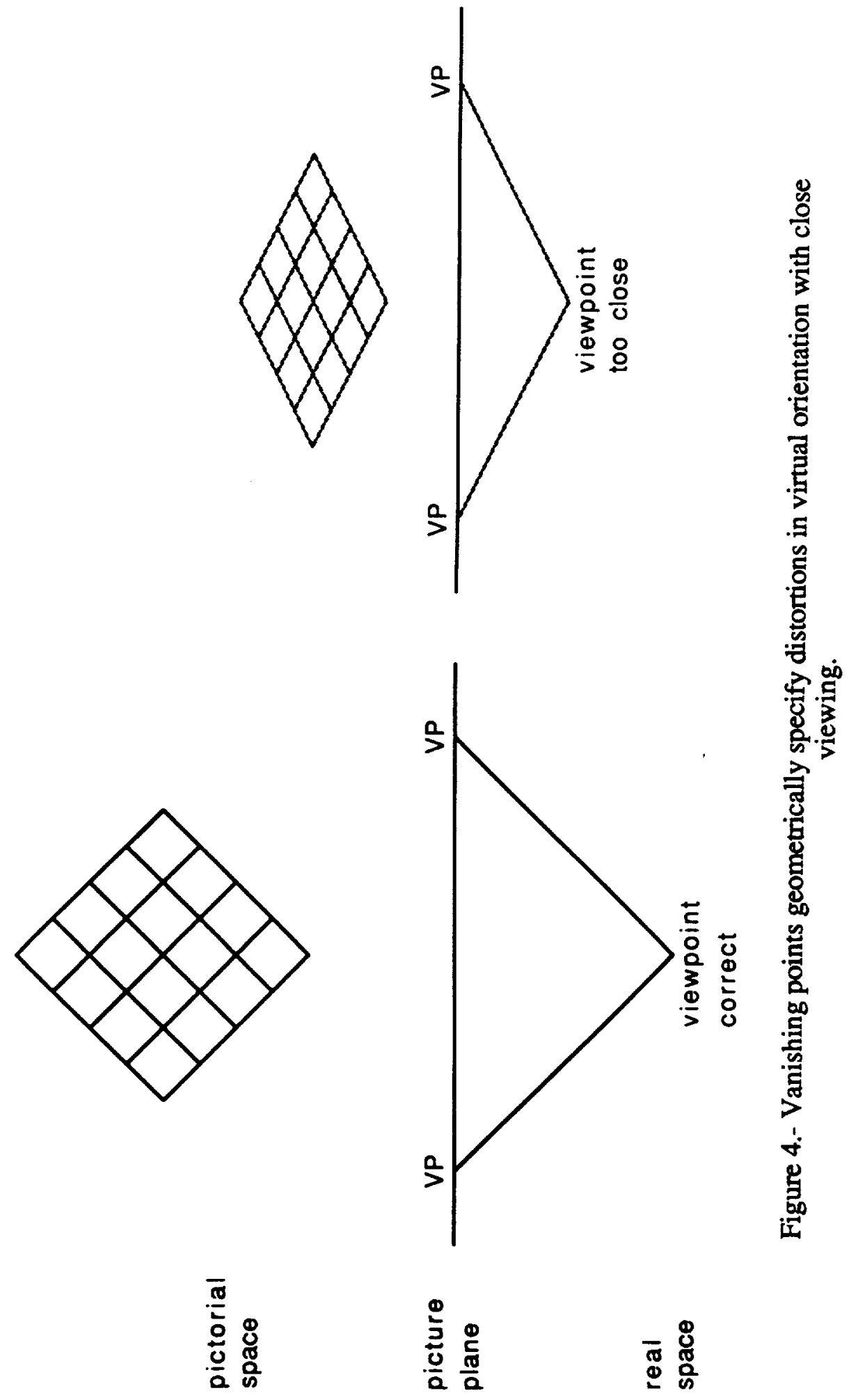




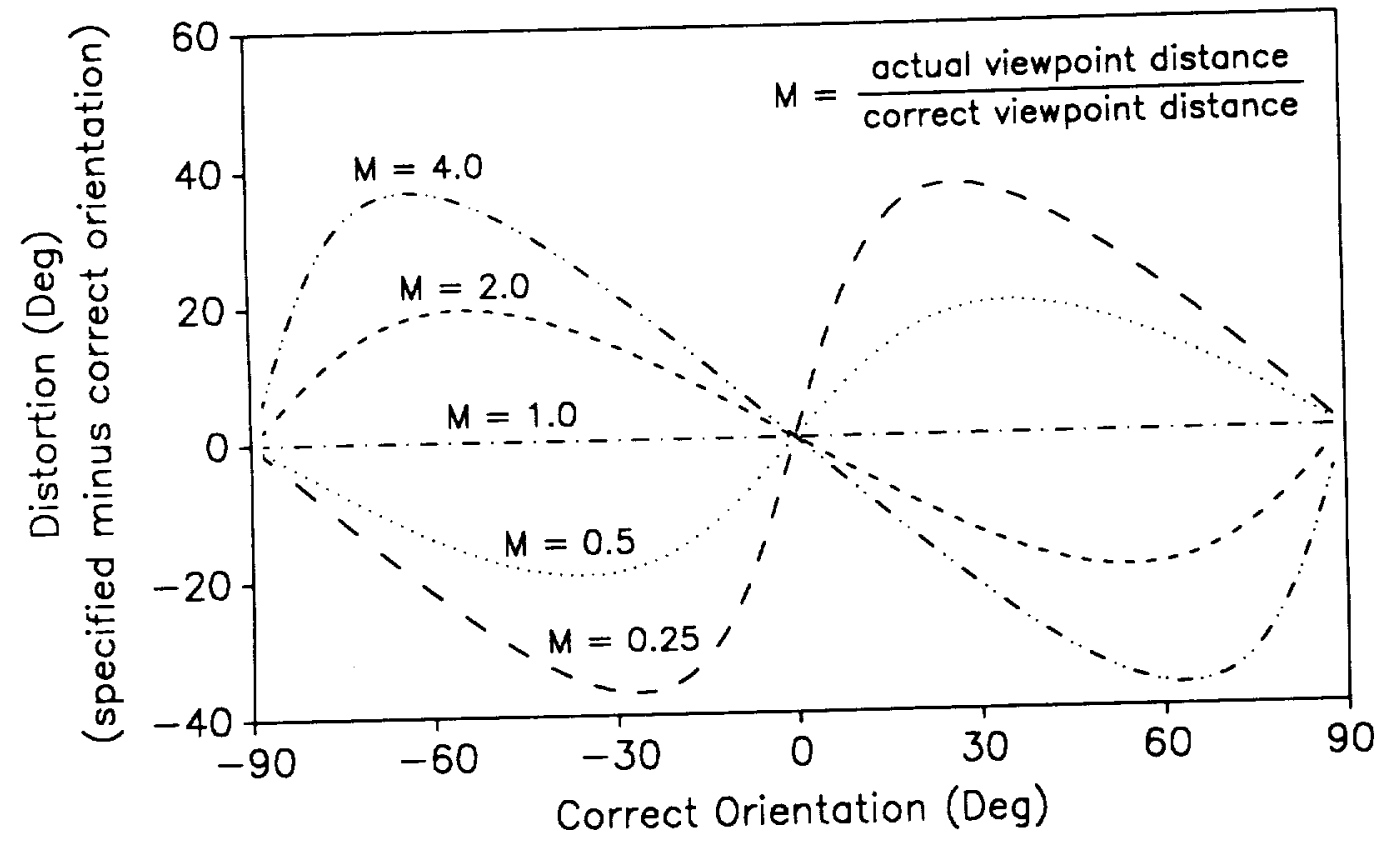

Figure 5.- Geometrically specified distortion in virtual orientation as a function of viewing distance.

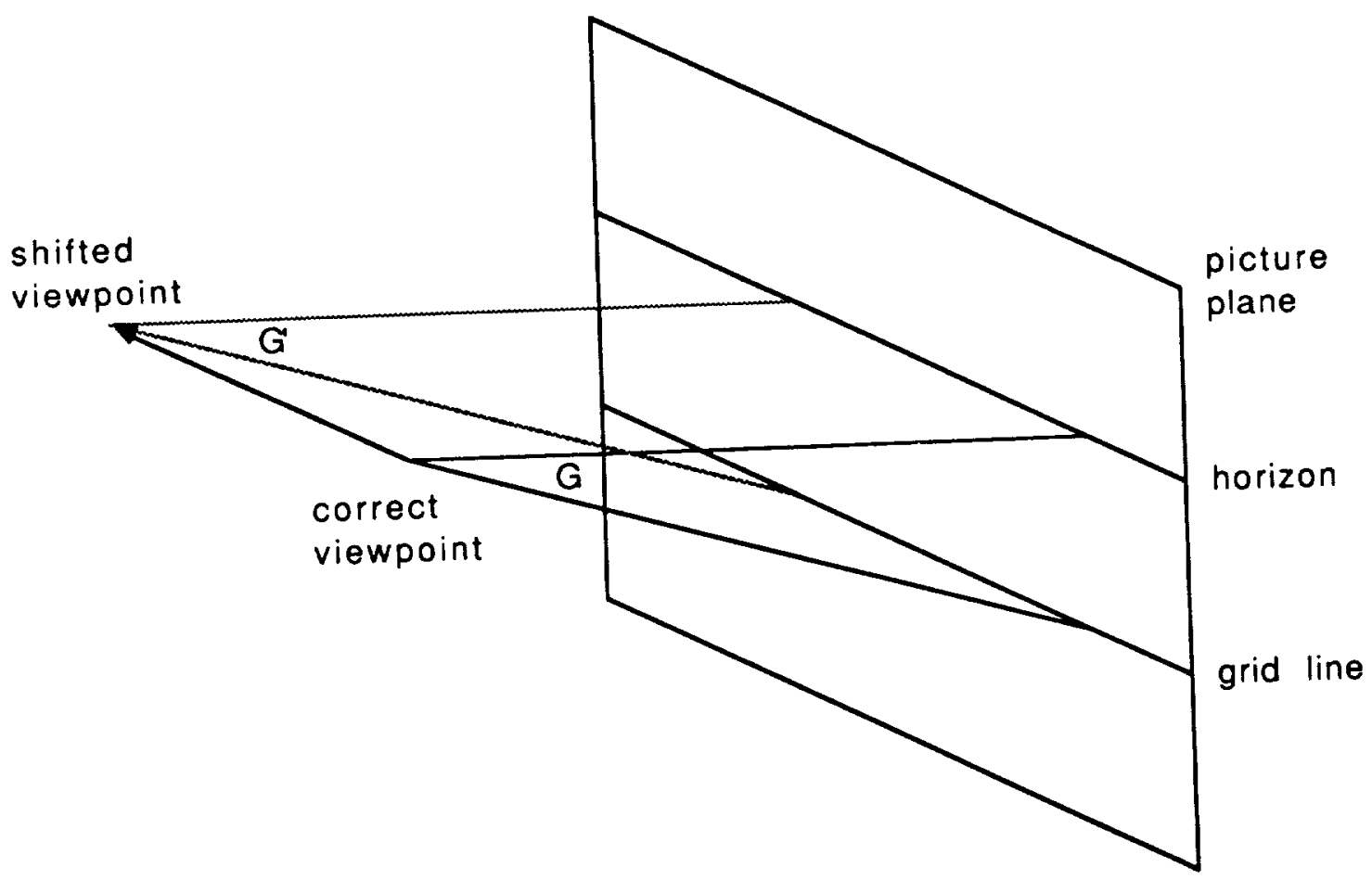

Figure 6.- Lateral shifts in viewpoint do not change geometrically specified virtual depth. 


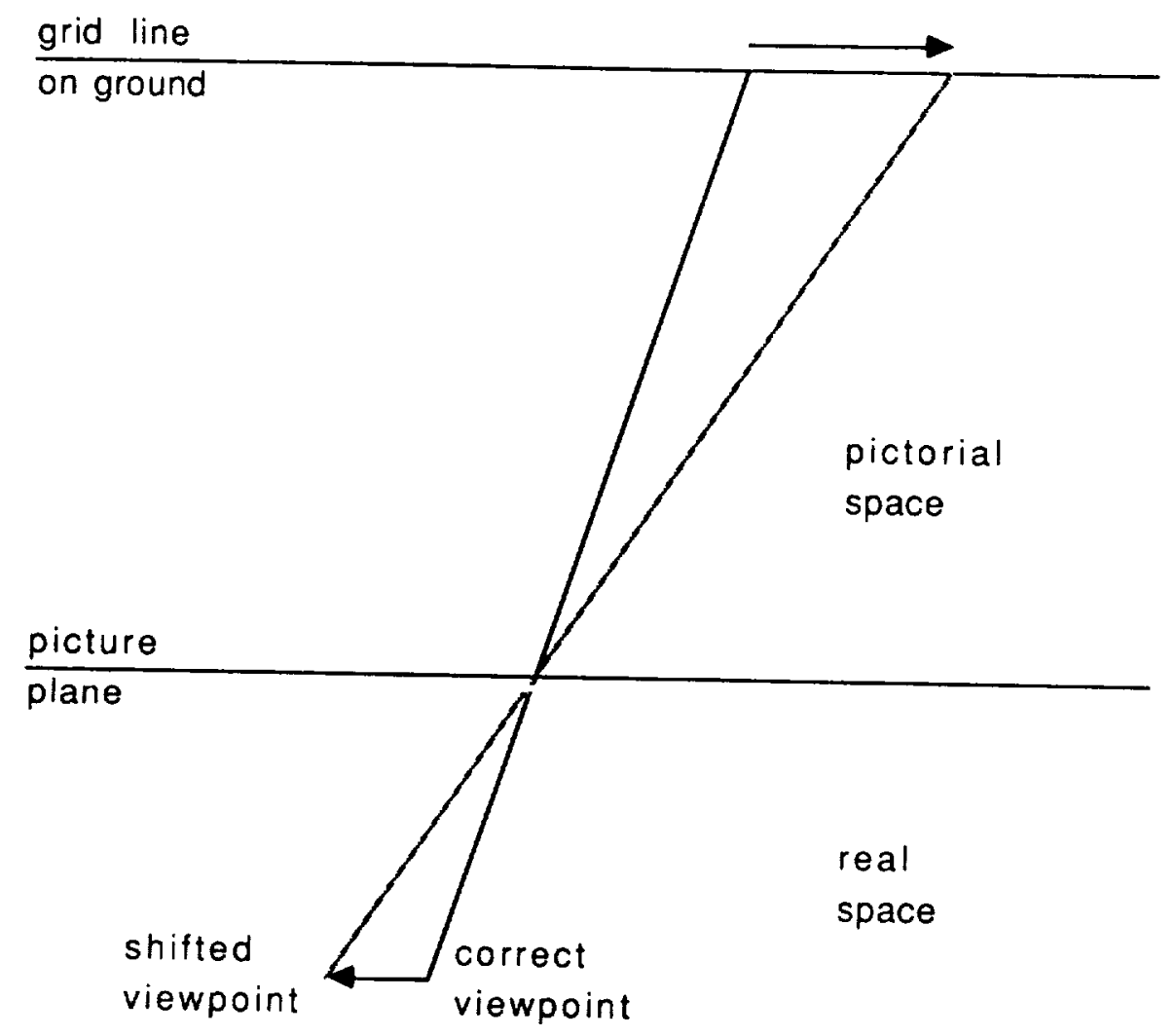

Figure 7.- Lateral shifts in viewpoint geometrically specify lateral shifts in virtual space. 


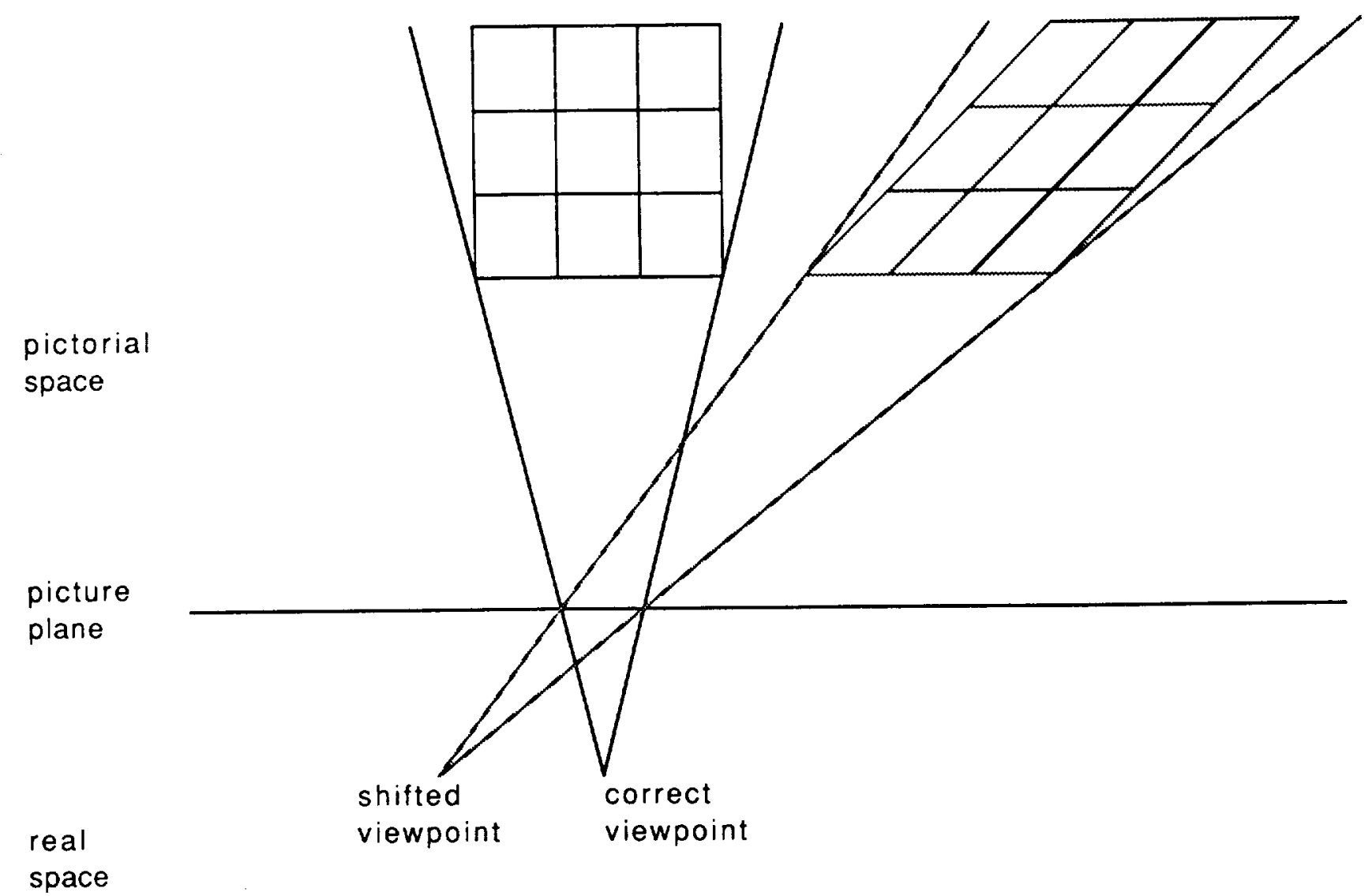

Figure 8.- Lateral shifts in viewpoint geometrically specify a shearing of virtual space. 


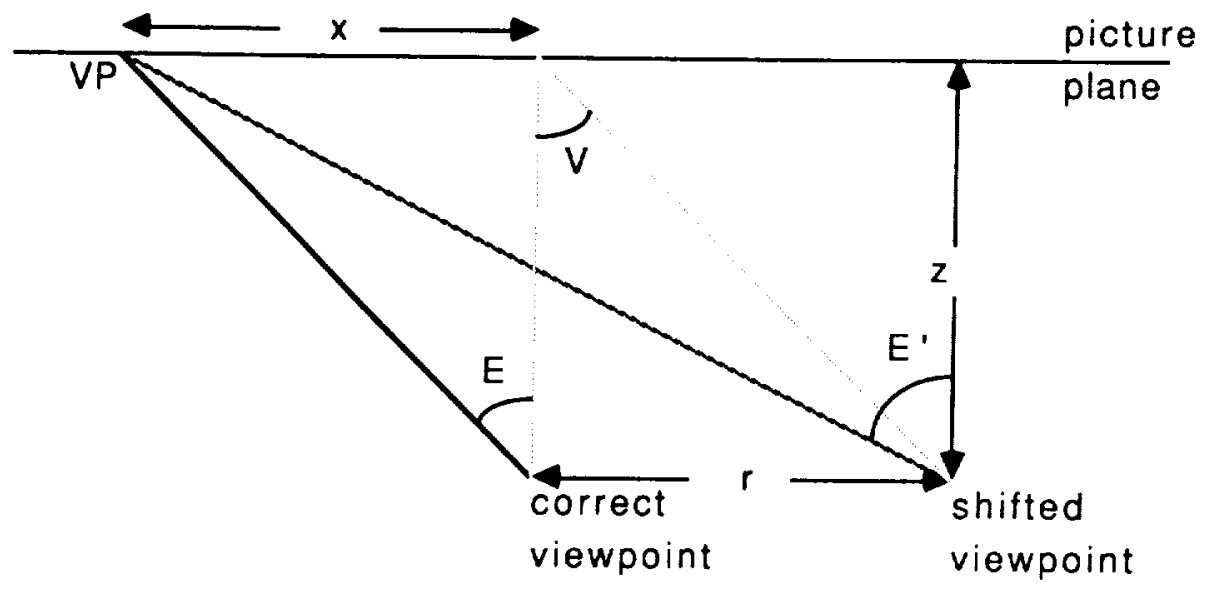

Figure 9.- Vanishing points geometrically specify distortions in virtual orientation with lateral shifts in viewpoint.

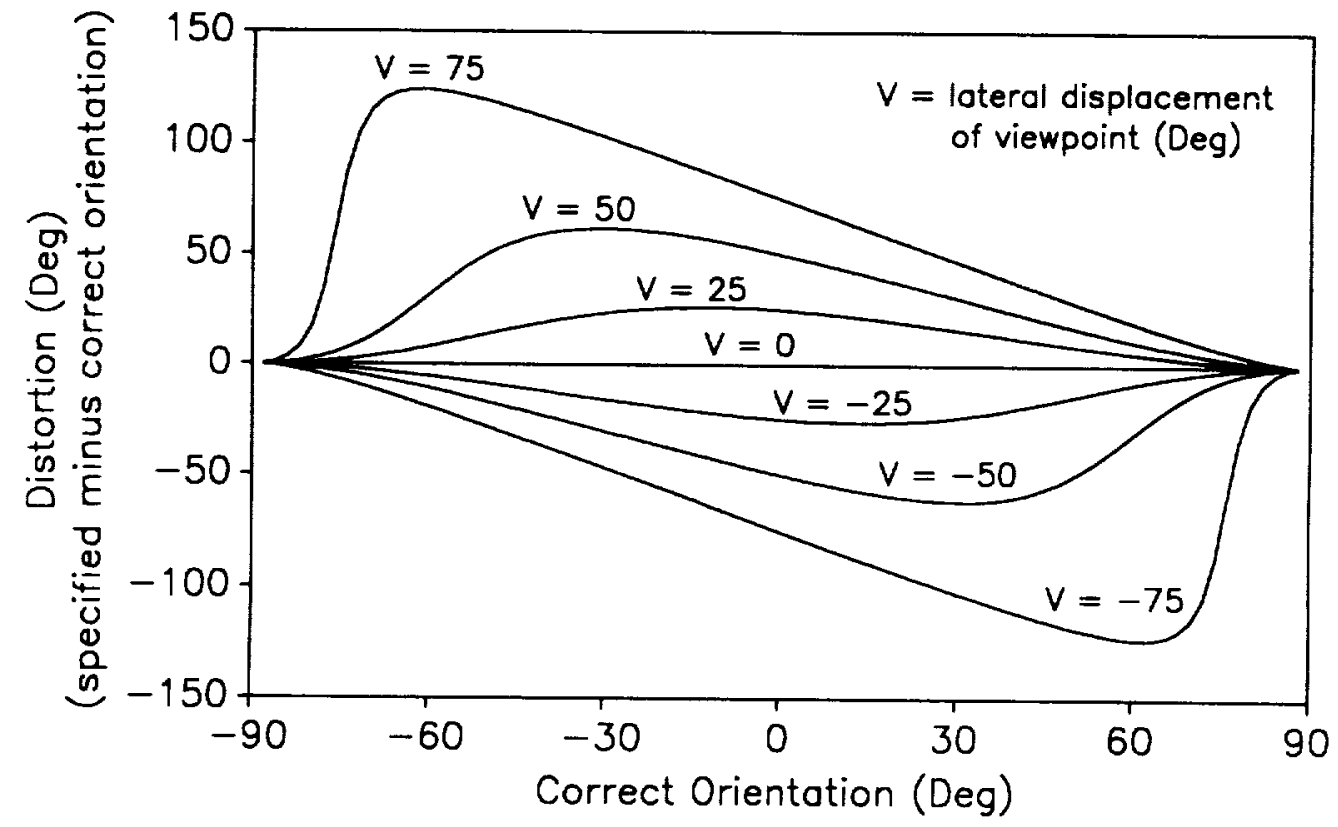

Figure 10.- Geometrically specified distortion in virtual orientation as a function of lateral shift in viewpoint. 